\title{
Síndrome de Fahr en un paciente pediátrico. Reporte de caso
}

Fahr syndrome in a pediatric patient. Case report

Aguilar Pesantez Martina de Jesús ${ }^{1}$, Cabrera Contreras Guadalupe Priscila²,Ochoa Arévalo Victor Francisco ${ }^{3}$

VOLUMEN 38 | Nº 3 | DICIEMBRE 2020

FECHA DE RECEPCIÓN: 17/11/2020 FECHA APROBACIÓN: 01/04/2021 FECHA DE PUBLICACIÓN: 06/04/2021

1. Ministerio de Salud Pública. Centro de Rehabilitación Integral Especializado. Departamento de Enfermería. Cuenca - Ecuador

2. Enfermera en libre ejercicio Cuenca - Ecuador

3. Hospital Monte Sinaí. Unidad de Pediatría

Cuenca - Ecuador

\begin{tabular}{c|c} 
Caso & Clinical \\
Clínico & Case
\end{tabular}

DOI: https://doi.org/10.18537/RFCM.38.03.09

Correspondencia:

martina.aguilar@ucuenca.edu.ec

Dirección:

Av. Roma y París

Código Postal:

010108

Teléfono:

4102194 - 0991657875

Cuenca - Ecuador

\section{RESUMEN}

Introducción: el Síndrome de Fahr es una ferrocalcinosis cerebrovascular idiopática, que provoca depósitos calcificados anormales en ganglios basales bilaterales, núcleos dentados, cerebelo y sustancia blanca cerebral, está asociado a síntomas neuropsiquiátricos. Es más frecuente en la edad adulta; sin embargo, puede presentarse con menor frecuencia en la etapa infantil. El presente artículo expone un caso de encefalopatía progresiva de inicio en la infancia.

Caso clínico: paciente masculino de 13 años, con episodios de estatus epilépticos recurrentes, movimientos anormales de la marcha y déficit cognitivo de varios años de evolución. En la Tomagrafía Axial Computarizada (TAC) se observóatrofia cortical difusa, calcificaciones groseras y simétricas de los núcleos de la base: cabeza de núcleo caudado, globus pallidus, calcificaciones tenues en los hemisferios cerebelosos. Imágenes compatibles con el Síndrome de Fahr.

Conclusiones: el Síndrome de Fahr puede ser una causa de crisis convulsivas y debe considerarse en la valoración clínica de un paciente con convulsiones, en especial en niños y adolescentes.

Palabras clave: calcinosis, ganglios basales, enfermedades del sistema nervioso, adolescente, TAC. 


\section{ABSTRACT}

Introduction: Fahr syndrome is an idiopathic cerebrovascular ferrocalcinosis, which causes abnormal calcified deposits in bilateral basal ganglia, dentate nuclei, cerebellum and cerebral white matter; it is associated with neuropsychiatric symptoms. It is more common in adulthood; however it can occur less frequently in childhood. This article presents a case of progressive encephalopathy that began in childhood.

Clinical case: a 13-year-old male patient, with recurrent status epileptic episodes, abnormal gait movements and cognitive deficit of several years of evolution. Diffuse cortical atrophy, coarse and symmetrical calcifications of the base nuclei: head of the caudate nucleus, globus palidus, and tenuous calcifications in the cerebellar hemispheres. Images are compatible with Fahr syndrome.

Conclusions: Fahr syndrome can be a cause of seizures and should be considered in the clinical evaluation of a patient with seizures, especially in children and adolescents.

Key words: calcinosis, basal ganglia, nervous system diseases, adolescent, TAC.

\section{INTRODUCCIÓN}

El Síndrome de Fahr es un trastorno neurológico raro caracterizado por depósitos de calcio en los ganglios basales, núcleos dentados, sustancia blanca y corteza cerebral. Comúnmente afecta a adultos jóvenes entre la tercera y cuarta década de vida ${ }^{1}$. Esta patología corresponde a una afección secundaria a causas orgánicas identificadas tales como: enfermedades endocrinas, autoinmunes, metabólicas, etc., y se caracteriza por el depósito anormal de calcio en ganglios basales (globus pallidus, putamen, núcleo caudado), núcleo dentado del cerebelo, tálamo, sustancia blanca subcortical y cerebelo, afecta por igual a ambos sexos ${ }^{2}$.
La prevalencia del Síndrome de Fahr es incierta; sin embargo, las calcificaciones cerebrales características de éste Síndrome se llegan a detectar de forma incidental en alrededor del $0.3 \%$ al $1.2 \%$ de las imágenes de TAC cerebral realizadas por otras razones ${ }^{3}$. Estudios indican que la mayoría de las calcificaciones se producen de forma bilateral y simétrica, una minoría se presenta de forma unilateral y sin la presencia de anomalías en el metabolismo del calcio ${ }^{4}$.

Las calcificaciones en sumayoría se encuentran en los ganglios basales, ya que son zonas de mayor acúmulo de sustancias como calcio, magnesio, fósforo entre otras, lo cual dificulta una adecuada perfusión cerebral y genera una respuesta inflamatoria local, afectando tanto los astrocitos como las neuronas agrupadas en los núcleos de sustancia gris basales ${ }^{5}$.

Los núcleos de la base, regulan el control del movimiento y el plan motor de acción con su ejecución, la presencia de calcificaciones en los núcleos de la base van a producir una amplia sintomatología neurológica asociada a la movilidad extrapiramidal ${ }^{6}$.

El término síndrome y enfermedad de Fahr han sido utilizados para describir distintas condiciones clínicas que involucran la calcificación de los núcleos de la base, pero es importante distinguir estas terminologías entre ellas. El Síndrome de Fahr hace relación a las calcificaciones de los núcleos de la base sin tener en cuenta la etiología y se considera secundaria en:

a. Anormalidades en el metabolismo del calcio y fosfato, principalmente hiper o hipo y pseudo-hipoparatiroidismo

b. Infecciones cerebrales, como el síndrome de inmunodeficiencia adquirida (SIDA), el virus de Epstein-Barr y otras meningoencefalitis. 
c. Tumores (principalmente angiomas y gliomas cerebrales).

d. Lupus eritematoso sistémico.

e. Traumatismos craneales previos.

La enfermedad de Fahr se usa para referirse a los trastornos primarios que presentan un mecanismo de herencia autosómico dominante y menos frecuente autosómico recesivo. Se describen diferentes mutaciones; la mutación en el gen SLC20A2 en el cromosoma 8p11 (OMIM\# 213600) fue la primera encontrada, también localizado en el locus IBGC1cromosoma 14 y cromosoma 2. Hasta el momento se han informado otras 5 mutaciones ${ }^{4,7-9}$.

La historia clínica, el examen y los hallazgos de laboratorio son cruciales para el diagnóstico diferencial. Es posible identificar formas secundarias asociadas con trastornos endocrinos. La TAC sigue siendo una de las técnicas más importantes para el diagnóstico del Síndrome de Fahr, permitiendo mayor especificidad ya que esclarece la causa de aparición de los síntomas a la hora de establecer un diagnóstico diferencial, evidenciando las calcificaciones bilaterales y simétricas de los tálamos, sustancia blanca, ganglios basales y cerebelo?.

En la mayoría de los casos de Síndrome de Fahr no existen antecedentes de tipo genético se observan calcificaciones bilaterales de los ganglios basales secundarios a trastornos del metabolismo del calcio y fósforo, especialmente hipoparatiroidismo. Existen tres tipos de anomalías bioquímicas de la homeostasis del fosfato de calcio: hipoparatiroidismo verdadero, pseudohipoparatiroidismo y las calcificaciones coexisten con los niveles séricos normales de calcio y fosfato ${ }^{10}$.
La etiología más frecuente del síndrome de Fahr, conjuntamente con la idiopática y las infecciones congénitas (toxoplasmosis) son las alteraciones del metabolismo del calcio, principalmente el hipoparatiroidismo primario y el pseudohipoparatirodismo, produciendo éstos hasta el $80 \%$ de los casos de calcificaciones de los ganglios basales ${ }^{11}$.

El síndrome de Fahr es más frecuente en la edad media y se caracteriza clínicamente por alteración del tono muscular y de la regulación de los movimientos voluntarios y automáticos, teniendo en cuenta que, desde un punto de vista clínico, el síndrome se manifiesta a través de sintomatología relacionada con disfunción del sistema extrapiramidal, como: movimientos atetósicos, distonías; déficit cognitivo; trastornos de conducta y en ocasiones puede cursar con crisis convulsivas. El signo característico en la imagenología cerebral, es la presencia de calcificaciones bilaterales y simétricas, en los núcleos de la base o en otras áreas cerebrales ${ }^{12}$. El interés de los autores en el estudio se basa fundamentalmente en considerar el hecho que en el país no se han reportado casos similares a temprana edad.

\section{PRESENTACIÓN DEL CASO}

Paciente de 13 años de edad, sexo masculino, etnia mestiza, estudiante, procedencia y residencia Huaquillas. Acude en compañía de su madre quien refiere que el niño hasta antes de cumplir los 8 años no tenía ninguna dificultad en su aprendizaje y realizaba de manera independiente todas las actividades de la vida diaria. A partir de esa edad el paciente comienza a presentar: crisis convulsivas (con fenomenología focal, motora, con alteración de la conciencia); dificultades en el aprendizaje, con fracaso escolar, sin poder acceder a los aprendizajes básicos del dominio de la lectura y escritura. En medio escolar se elabora 
un programa individualizado que responda a sus necesidades educativas especiales; sin embargo, los escasos conocimientos académicos adquiridos se pierden, porque las crisis se volvieron de difícil control. A los 10 años abandonasusestudiosporsu condición desalud y deterioro en sus conductas de interacción social con sus pares, con autoaislamiento, dificultades en su comunicación verbal y no verbal; autoagresiones y heteroagresiones. No refiere consumo de alcohol, tabaco ni otras drogas.

El paciente fue ingresado en el hospital Teófilo Dávila en octubre del 2017, por estatus convulsivo, fármaco anticonvulsivante prescrito al egreso hospitalario; las crisis convulsivas persisten activas, con una frecuencia de 5-7 crisis diarias; razón por la que su madre acude el 27 de noviembre de 2017 al CRIE No 5 (Centro de Rehabilitación Integral Especializado número 5).

Paciente fruto de séptima gestación, edad materna a la gestación 35 años, embarazo sin complicaciones aparentes, período natal y post natal normal; hitos del neurodesarrollo normal. Refiere fallecimiento de hermano al año de edad y no se estableció causa de muerte. Alos 8 años de edad, se inician crisis convulsivas, en un contexto de deterioro neurológico, evidente en su motricidad global, marcha tambaleante, con caídas frecuentes, presencia de movimientos involuntarios bruscos. Igualmente deterioro en sus habilidades de aprendizaje académico, con retroceso de estos aprendizajes y dificultades en sus conductas adaptativas social con sus pares, por alejamiento social y agresividad.

Al examen físico: frecuencia respiratoria 22, frecuencia cardíaca: 102, saturación de oxígeno 90\%. Datos antropométricos peso: $25.2 \mathrm{~kg}$ (bajo percentil - 3) Talla: $124 \mathrm{~cm}$ (bajo percentil -3); examen general: normal.
Examen neurológico: Estado de conciencia: orientado.

Motricidad global: el paciente se desplaza con ayuda de un adulto, con patrón de bipedestación y desplazamiento atáxico, que no se agrava al cerrar los ojos, aumento de la base de sustentación, dificultades en la equilibración unipodal; dificultad en la ejecución de movimientos alternos con sus extremidades, discronometría y dismetría. Pares craneales: nistagmos y sacadas visoguiadas en el eje horizontal. Reflejo osteotendinosos disminuidos en sus cuatro extremidades. Perfil cognitivo: no responde a baterías psicométricas estándar para su edad. Se aplica pruebas piagetianas, donde alcanza una edad de razonamiento de 2 años 6 meses.

Exámenes: TAC se observa atrofia cortical, calcificación simétrica de los núcleos de la base: cabeza de núcleo caudal, globus pallidus y calcificaciones tenues en los hemisferios cerebelos, imágenes compatibles con el síndrome de Fahr (ver imagen $N^{\circ} 1$ ). Serie ósea normal.

Exámenes de laboratorio: hormonas TSH 1.93 $\mathrm{Uu} / \mathrm{ml}$ normal y FT4 1.04ng/dL, discretamente bajo, hormona paratiroidea $14.46 \mathrm{pq} / \mathrm{ml}$ por debajo del rango normal; hormona de crecimiento IGF $1230.00 \mathrm{ng} / \mathrm{ml}$ normal y IGF 3 con un valor de $1900 \mathrm{ng} / \mathrm{ml}$ por debajo del rango normal; calcio, fósforo, fosfatasa alcalina normal (ver Tabla $N^{\circ} 1$ ). En la escala de comportamiento de Corrigan: perfil desinhibición en el 65\%; agresividad 41\%; labilidad 25\%. 

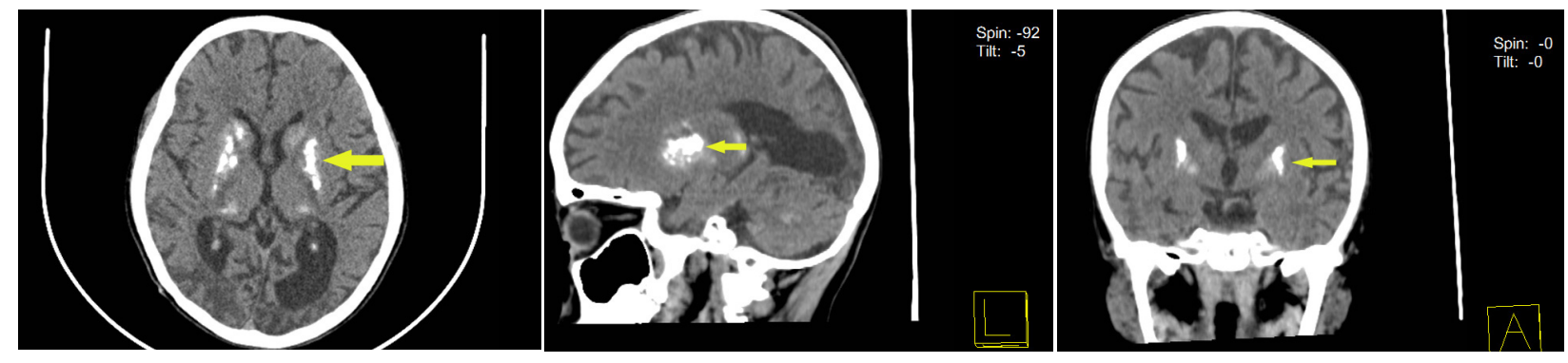

Imagen $\mathrm{N}^{\circ} 1$

Tomografía computada multicortes del cráneo. Calcificaciones bilaterales en los ganglios basales y espacios periventriculares

\section{Tabla $\mathrm{N}^{\circ} 1$}

Resultados de laboratorio y valores de referencia

\begin{tabular}{lcc}
\hline \multicolumn{1}{c}{ Prueba } & Resultado & Valor de referencia \\
\hline Calcio total & $9.3 \mathrm{mg} / \mathrm{dl}$ & $8.40-10.20 \mathrm{mg} / \mathrm{dl}$ \\
Fósforo & $4.8 \mathrm{mg} / \mathrm{dl}$ & $2.5-5 \mathrm{mg} / \mathrm{dl}$ \\
Fosfatasa alcalina & $163 \mathrm{U} / \mathrm{l}$ & $0390 \mathrm{U} / \mathrm{l}$ \\
Creatinina & $0.52 \mathrm{mg} / \mathrm{dl}$ & $0.701 .20 \mathrm{mg} / \mathrm{dl}$ \\
Hormona paratiroidea & $14.46 \mathrm{pg} / \mathrm{ml}$ & $16-87 \mathrm{pg} / \mathrm{ml}$ \\
\hline
\end{tabular}

Para las crisis convulsivas se prescribió ácido valproico y carbamazepina, se recomienda complementar con estudio genético. En el control neurológico realizado a los cinco meses se determina que no hay mejoría de su sintomatología y por el contrario se comprueba mayor deterioro cognitivo, por lo cual familiares del paciente deciden abandonar la institución y no se ha podido realizar un seguimiento adecuado debido a la ubicación geográfica, las condiciones socioeconómicas de la familia, con deterioro de la salud, paciente fallece.

\section{DISCUSIÓN}

El síndrome de Fahr es una patología poco común y de baja incidencia, siendo muy pocos los casos descritos en edades pediátricas. Según la revisión bibliográfica no se obtuvo mayor información y es muy limitado el estudio comparativo a nivel mundial, también cabe recalcar que existen discrepancias entre el síndrome y la enfermedad de Fahr debido a la similitud de la sintomatología. La enfermedad de Fahr se refiere a la calcificación familiar primaria de los ganglios basales, mientras que en el síndrome de Fahr las calcificaciones son secundarias, esto se debe a otros contextos asociados con el metabolismo anormal del calcio y el fosfato ${ }^{13}$.

Las manifestaciones clínicas de este síndrome incorporan una amplia variedad de síntomas, que van desde síntomas neurológicos del sistema extrapiramidal a anormalidades neuropsiquiátricas de la memoria y la concentración; a trastornos del movimiento que incluyen parkinsonismo, corea y temblores, otras como trastornos del habla, crisis convulsivas y deterioro cognitivo; estos dos últimos fueron la presentación del caso.

No se demostró trastornos del metabolismo del calcio, se manifiesta los síntomas secundarios a un proceso neurodegenerativo encefálico 
debido a un hipotiroidismo subclínico ${ }^{4}$. El signo característico en la imagenología cerebral, es la presencia de calcificaciones bilaterales y simétricas, en losnúcleos de labase, hemisferios cerebelosos e imágenes hipodensas en la sustancia blanca periventricular con evidencias de retracción de las astas posteriores de los ventrículos laterales. La TAC es fundamental para confirmar el diagnóstico del síndrome de Fahr y descartar otras entidades.

No existe un tratamiento actual para el síndrome de Fahr, los tratamientos están dirigidos a minimizar los síntomas. Es por esto que el pronóstico para las personas con síndrome de Fahr es poco alentador ya que existe deterioro neurológico progresivo. En la mayoría de los casos, el tratamiento farmacológico es sintomático e incluye antipsicóticos, antidepresivos, fármacos antiepilépticos ${ }^{10,12,14}$. Como otra forma de tratamiento se podría considerar la rehabilitación física y ocupacional que conlleva a disminuir, mantener y mejorar de cierta manera las condiciones motrices y mejorar la calidad de vida dándole confort en el proceso de la enfermedad cuyo procedimiento está enfocado al control postural, fortalecimiento muscular, arcos de movimiento y patrón de marcha.

En el estudio de Ortiz y Mendoza ${ }^{14}$ en 2020, sobre el síndrome de Fahr se encontró la siguiente sintomatología en el paciente: movimientos distónicos cervicales y de miembros superiores sospechándose que eran de origen convulsivo, en las imágenes se evidenciaron hiperdensidades en ganglios basales y subcorticales bilaterales, hipocalcemia e hiperfosfatemia, paratohormona baja. En cambio, en el estudio de caso detectado en el CRIE N 5, se encontró que el paciente en las pruebas bioquímicas no presenta hipocalcemia ni hiperfosfatemia, pero si presenta paratohormona baja, crisis convulsivas, signos extrapiramidales y síntomas neuropsiquiatricos.

En el estudio de Manea y Sirbu ${ }^{13}$ en 2020, sobre convulsiones epilépticas como primera manifestación del síndrome de Fahr en un paciente de 24 años encontrándose la siguiente sintomatología: convulsiones tónico-clónicas generalizadas que aumentaron en frecuencia durante el último año, la TAC realizada al ingreso mostró depósitos de calcio en núcleos dentados, ganglios basales, tálamo, cerebelo y sustancia blanca, los resultados de las pruebas de laboratorio fueron normales a excepción de hipocalcemia, hiperfosforemia y parathormona intacta baja. En el caso detectado en el CRIE N05 se encontró similitud en relación a las convulsiones que se incrementaron el último año, las calcificaciones detectadas por TAC y en las pruebas de laboratorio en especial la parathormona discretamente baja.

En varios estudios realizados por diferentes autores se establecen criterios diagnósticos para el síndrome de Fahr ${ }^{10}$, siendo los siguientes:

1. Disfunción neurológica progresiva que incluye desórdenes del movimiento y manifestaciones neuropsiquiátricas.

2. La edad de inicio es típicamente hacia cuarta o quinta década de la vida; sin embargo, la disfunción puede estar presente en niños o en las décadas avanzadas de la vida.

3. Calcificaciones bilaterales de los ganglios basales $u$ otras regiones del cerebro que incluyen el cerebelo, el tallo cerebral, el centro semioval, y la sustancia blanca subcortical que se visualizan en la neuroimagen.

4. Ausencia de anormalidades bioquímicas o características somáticas sugestivas 
de una enfermedad metabólica o mitocondrial u otro desorden sistémico.

5. Ausencia de una causa infecciosa, tóxica o traumática.

6. Historia familiar consistente con una herencia autosómica dominante.

7. Calcificaciones visualizadas en la TAC y que afecten bilateralmente el strio-pallido-dentate.

En el caso detectado en el CRIE $N^{\circ} 5$, el paciente cumple con alguno de los criterios diagnósticos.

En el país no se han registrado otros casos en edad infantil siendo el estudio, único y particular. Se presentaron algunas limitaciones como el poco interés por parte de la familia para realizar tratamiento, básicamente por su condición socio-económica y a la distancia entre el CRIE N 5 y el lugar de residencia.

\section{CONCLUSIONES}

En el síndrome de Fahr pueden coexistir muchos síntomas que se asemejan a otras enfermedades neurológicas, psiquiátricas y cardiológicas, por eso es necesario diferenciar los casos de acuerdo a la clínica y exámenes complementarios. La TAC sigue siendo el método principal para el diagnóstico de síndrome de Fahr.

Al considerarse esta afección de etiología idiopática y de aparición en edades tempranas, con síntomas clínicos neurológicos progresivos y neuropsiquiátricos, debe tenerse en cuenta al momento de realizar el diagnóstico diferencial, de movimientos anormales en edades pediátricas, no solo en la búsqueda intencionada de calcificaciones sino para descartar otras patologías que pudieran presentar los mismos síntomas. Este caso clínico debe despertar el interés en los equipos de salud, para detectar patologías raras, de carácter evolutivo, que requieren un abordaje multiprofesional.

\section{ABREVIATURAS}

TAC: Tomografía axial computarizada

MRI: Imagen por resonancia magnética

CRIE: Centro de Rehabilitación Integral Especializado

\section{ASPECTOS BIOÉTICOS}

El presente estudio se efectuó bajo total confidencialidad de datos personales del paciente y con el respectivo consentimiento informado.

Perspectiva del paciente: Madre del niño refiere que se encuentra en peores condiciones y se comprueba mayor deterioro cognitivo.

\section{INFORMACIÓN DE LOS AUTORES}

Aguilar Pesántez Martina de Jesús. Licenciada en Enfermería. Magister en Gestión en Atención Primaria de Salud. Ministerio de Salud Pública. Centro de Rehabilitación Integral Especializado. Departamento de Enfermería. Cuenca - Azuay Ecuador.

e-mail: martina.aguilar@ucuenca.edu.ec

ORCID:https://orcid.org/0000-0001-8122-7079

Cabrera Contreras Guadalupe Priscila. Licenciada en Enfermería. Magister en Gestión en Atención Primaria de Salud. Libre ejercicio. Cuenca - Azuay - Ecuador.

e-mail: lupita.cabrera0785@gmail.com

ORCID:https://orcid.org/0000-0002-9232-0049

Ochoa Arévalo Víctor Francisco. Doctor en Medicina y Cirugía. Magister en Neuropsicología Infantil. Hospital Monte Sinaí. Unidad de Pediatría. Cuenca - Azuay - Ecuador.

e-mail: pacoochoacdt@msn.com

ORCID:https://orcid.org/0000-0003-4258-8167 


\section{CONTRIBUCIÓN DE LOS AUTORES}

APMJ, CCGP: concepción y diseño del estudio, recolección de datos, redacción y revisión del presente artículo.

OAVF: concepción del estudio, redacción y revisión del presente artículo.

\section{CONFLICTO DE INTERESES}

Los autores declaran no tener conflicto de intereses.

\section{FUENTES DE FINANCIAMIENTO}

Fondos propios.

\section{REFERENCIAS BIBLIOGRÁFICAS}

1. Saleem $S$; Muhammad $H$; Anwar $M$; Anwar S; Saleem M; Saleem A; et al. Fahr's syndrome: Literature review of current evidence. Orphanet J Rare Dis [Internet]. 2013;8(1):1-9. Available from: http://www. ojrd.com/content/8/1/156\%0A

2. SucreFM; Casares K; Sandoval J. Calcinosis bilateral en los núcleos estriado, pálido y dentado e hipoparatiroidismo: a propósito de un caso. Rev An Radiol Mex [Internet]. 2015;14:285-91. Available from: https:// www.medigraphic.com/pdfs/anaradmex/ arm-2015/arm153f.pdf

3. Ooi H, Er C, Hussain I, Kuthiah N A V. Bilateral Basal Ganglia Calcification: Fahr' $S$ Disease Case Presentation. Cureus [Internet]. 2019;11(6):1-8. Available from: $\quad$ https://www.researchgate.net/ publication/333550593\%0D

4. Pistacchi M; Gioulis M; Sanson F; Zambito S. Fahr's syndrome and clinical correlation: A case series and literature review. Folia Neuropathol. 2016;54(3):282-94. Available from: https://www.termedia.pl/Fahr-s- syndrome-and-clinical-correlation-a-caseseries-and-literature-review,20,28391,1,1. html

5. Van S; Severino M; Ammendola R; Van B; Vavro $\mathrm{H}$; Van L. et al. Bilateral lesions of the basal ganglia and thalami (central grey matter)-pictorial review. Neuroradiology [Internet]. 2020;62(12):1565-605. Available from: https://doi.org/10.1007/s00234-02002511-y\%0A

6. Avila A; Bueno A. Los ganglios basales: la participación dopaminérgica estriatal. Investig en Discapac [Internet]. 2014;3(1):19-24. Available from: https:// www.medigraphic.com/pdfs/invdis/ir2014/ir141c.pdf

7. Lacoma E; Sánchez E; Rubio P. Enfermedad de Fahr. Imagen Diagnostica [Internet]. 2016;7(2):74-5. Available from: https:// www.elsevier.es/es-revista-imagendiagnostica-308-pdf-S2171366916000068

8. Savino E, Soavi C, Capatti E, Borrelli M, Vigna G, Passaro A, et al. Bilateral strio-pallidodentate calcinosis (Fahr's disease): report of seven cases and revision of literature. BMC Neurol [Internet]. 2016;16(1):165. Available from: doi: 10.1186/s12883-0160693-1

9. Mufaddel A A-HG. Familial idiopathic basal ganglia calcification (Fahr's disease). 2014;19(3):171-7. Available from: https:// www.ncbi.nlm.nih.gov/pmc/articles/ PMC4727649/

10. Jaworski K, Styczyńska Mandecka M, Walecki J, Kosior. Fahr syndrome An important piece of a puzzle in the differential diagnosis of many diseases. Polish J Radiol [Internet]. 2017;82:490-3. Available from: https://www.ncbi.nlm.nih. gov/pmc/articles/PMC5894054/ 
11. Nicolau N; Espino A; Rivera R; Frances C; Masmiquel L. Calcinosis estriado-pálidodentada como causa deextrapiramidalismo en una paciente con hipoparatiroidismo primario. 2012;59(1):69-71. Available from: https://www.elsevier.es/es-revistaendocrinologia-nutricion-12-articulocalcinosis-estriado-palido-dentadacomo-causa-extrapiramidalismo -S1575092211001987

12. Verbel L; Torres $\mathrm{M}$; Cabarcas $\mathrm{O}$; et al. Fahr disease an infrequent cause of brain calcifications Caso clínico. Acta Neurol [Internet]. 2011;27:5. Available from: http:// www.scielo.org.co/scielo.php?script=sci_ arttext\&pid=S0120-87482011000200006

13. Manea MM, Sirbu A, Dragos D, Dobri AM, Sirbu AG, Tuta S. Epileptic seizures as the first manifestation of fahr's syndrome. Acta Endocrinol (Buchar). 2020;16(3):370374. doi: 10.4183/aeb.2020.370.

14. Roa M; Mendoza V. Síndrome de Fahr secundario a hipoparatiroidismo: una causa infrecuente de movimientos anormales en niños. 2020;(July). Available from: https://www.researchgate.net/ publication/342961919_NOTA_CLINICA_ e164_Sindrome_de_Fahr_secundario_a_ hipoparatiroidismo_una_causa infrecuente_de_movimientos_anormales_ en_ninos 